\title{
Análise do equilíbrio espinopélvico em pacientes com espondilolistese degenerativa L4-L5
}

\author{
Elton Luiz Batista Cavalcante', Lucas Almeida Campagnaro', José Lucas \\ Batista Junior', Charbel Jacob Júnior², Igor Machado Cardoso²
}

Hospital Santa Casa de Misericórdia de Vitória, Vitória, ES, Brasil.

\section{RESUMO}

Objetivos: Avaliar o equilíbrio espinopélvico em pacientes portadores de espondilolistese degenerativa L4-L5. Métodos: Analisamos as radiografias pré-operatórias lombopélvicas, em perfil, de 33 pacientes portadores de espondilolistese degenerativa L4-L5 para a mensuração dos parâmetros envolvidos no equilíbrio espinopélvico. Resultados: Dos 33 pacientes, 22 pertencem ao sexo feminino, com média de idade de 59 anos. A média da incidência pélvica (IP), inclinação pélvica (TILT) e inclinação sacral (SLOP) foi $61,4^{\circ} ; 22,1^{\circ}$ e $38,3^{\circ}$, respectivamente, havendo correlação positiva entre IP e TILT e correlação negativa entre SLOP e TILT, ambas com significância estatística. Conclusão: Na espondilolistese degenerativa L4-L5, a IP, TILT e SLOP tiveram suas médias iguais a $61,4^{\circ}, 38,3^{\circ} \mathrm{e}$ $22,1^{\circ}$, respectivamente, revelando alteração na conformação pélvica que culminou em um desequilíbrio espinopélvico nesses pacientes.

\section{PALAVRAS-CHAVE}

Espondilolistese, osteoartrose, vértebras lombares.

\section{ABSTRACT}

Analysis of spinopelvic balance in patients with L4-L5 degenerative spondylolisthesis Objectives: To evaluate and analyze the spinopelvic balance in patients with L4-L5 degenerative spondylolisthesis. Methods: We analyzed the lumbopelvic preoperative radiographs in profile, 33 patients with L4-L5 degenerative spondylolisthesis to measure the parameters involved in spinopelvic balance as pelvic incidence (PI), pelvic tilt (TILT pelvic) and sacral slope (SLOP sacral). Results: Of the 33 patients with degenerative spondylolisthesis, 22 were female and the mean age was 59 years. The average IP, TILT and SLOP was $61.4^{\circ}, 22.1^{\circ}$ and $38.3^{\circ}$, respectively, with a positive correlation between IP and TILT and a negative correlation between SLOP and TILT, both with statistical significance. Conclusion: Patients with L4-L5 DS, IP, TILT and SLOP had their means equal to $61.4^{\circ}, 38.3^{\circ}$ and $22.1^{\circ}$, respectively, demonstrated that possibility the pelvic imbalance in these patients.

\section{KEYWORDS}

Spondylolisthesis, osteoarthritis, lumbar vertebrae.

1 Graduando do Curso de Medicina da Escola Superior de Ciências da Saúde da Santa Casa de Misericórdia de Vitória, Vitória, ES, Brasil.

2 Ortopedista e cirurgião da coluna, médico-assistente do Grupo de Coluna Vertebral do Hospital Santa Casa de Misericórdia de Vitória, Vitória, ES, Brasil. 


\section{Introdução}

A espondilolistese consiste no deslocamento anterior de uma vértebra sobre a subjacente, ocorre em função de um defeito ósseo na pars interarticularis ou na faceta vertebral e essas alterações facetárias podem ser de origem traumática, congênitas ou degenerativas. ${ }^{1-4}$

Dentre as diversas espondilolisteses, a do tipo III da classificação de Wiltse et al. ${ }^{5}$ consiste na espondilolistese degenerativa (ED), que ocorre devido a uma degeneração discal e facetária com arco vertebral íntegro, ${ }^{4,5}$ e acomete frequentemente o sexo feminino, na idade adulta ${ }^{6-9}$ e no nível de L4-L5. ${ }^{10,11}$ Esse tipo está comumente associado a graus variados de estenose do canal vertebral, sendo tida como causa frequente de lombalgia associada ou não a radiculopatia e claudicação intermitente. ${ }^{3,12}$

O balanço sagital e o equilíbrio espinopélvico vêm sendo cada vez mais estudados por ortopedistas, pois, por meio deles, podemos quantificar a origem de diversas patologias musculoesqueléticas. Normalmente eles atuam em conjunto levando a uma interação entre morfologia óssea (pélvica e vertebral), mecânica discal, resistência muscular e ligamentar, agindo como forças de atuação mútuas capazes de fornecer mecanismos compensatórios para manter a estabilidade corporal. ${ }^{13}$ Quando esse conjunto trabalha harmoniosamente e em equilíbrio, encontramos o ponto ideal para a marcha e consequentemente para o bem-estar das pessoas.

Apesar de existirem diversos trabalhos para análise do equilíbrio espinopélvico nas patologias ortopédicas, encontramos poucos estudos que analisassem a relação entre a ED e o equilíbrio espinopélvico. Julgamos que, por meio de uma melhor definição do equilíbrio espinopélvico nesses pacientes, poderemos realizar medidas preventivas para o não desenvolvimento ou progressão das listeses, bem como auxiliar no tratamento cirúrgico dos pacientes que não responderam positivamente ao tratamento conservador.

\section{Casuística e métodos}

Estudo retrospectivo, de caráter descritivo, a partir do levantamento de dados obtidos por meio de radiografias lombopélvicas em ortostase no período pré-operatório, realizado na Santa Casa de Misericórdia de Vitória/ES após aprovação do Comitê de Ética em Pesquisa sob $\mathrm{N}^{\circ} 231.213$. Foram avaliados 33 pacientes, dos quais 22 eram do sexo feminino, com a média de idade de 59,21 anos, variando entre 30 e 80 anos.

Foram incluídos neste trabalho pacientes portadores de ED L4-L5, classificados segundo os critérios de
Wiltse et al., ${ }^{5}$ de ambos os sexos, de qualquer idade, em período pré-operatório de descompressão medular e artrodese L4-L5, que fizeram acompanhamento ambulatorial no Hospital Santa Casa de Misericórdia de Vitória.

Foram excluídos os pacientes portadores de ED sem indicação cirúrgica, bem como pacientes que eventualmente apresentaram algum processo patológico associado à ED que dificulta a mensuração e avaliação do equilíbrio espinopélvico.

A mensuração dos parâmetros utilizados nesta pesquisa foi a incidência pélvica (IP), inclinação pélvica (TILT pélvico) e inclinação sacral (SLOP pélvico), sendo realizada rotineiramente em todos os pacientes com indicação cirúrgica de ED, a partir das radiografias pré-operatórias panorâmica da coluna vertebral e lombopélvicas em perfil.

Para a realização da mensuração angular utilizamos radiografia panorâmica da coluna vertebral no plano sagital, constituindo os chamados parâmetros pélvicos de Duval-Beaupère. A IP é um parâmetro morfológico-anatômico fixo, descrito como um ângulo subtendido por uma linha traçada a partir do centro das cabeças femorais para o ponto médio da placa terminal sacral (S1) e uma linha perpendicular ao ponto médio da placa sacral (S1) (Figura 1).

O TILT pélvico é um parâmetro posicional, formado pelo ângulo traçado entre a linha que liga o ponto médio da placa terminal do sacro (S1) ao eixo central da cabeça femoral e a linha de referência num plano vertical (Figura 2).

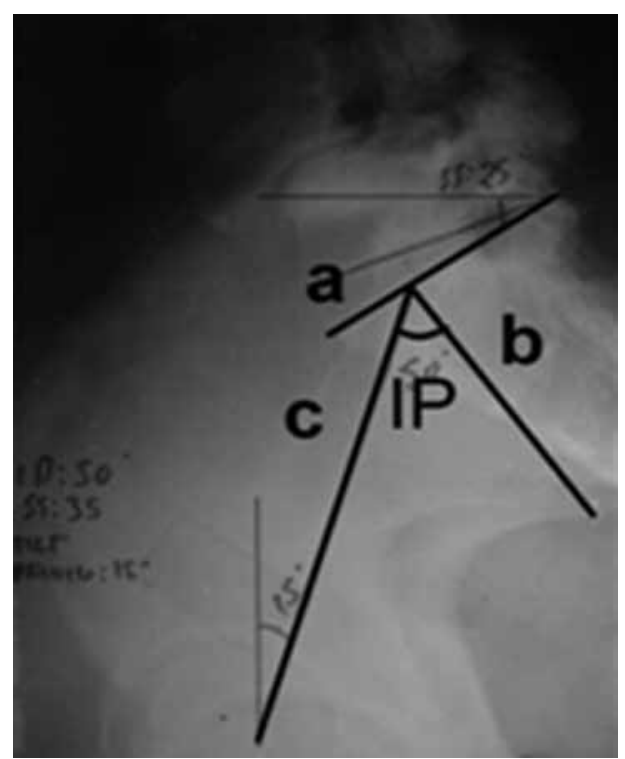

a: placa sacral;

b: linha perpendicular ao ponto médio da placa sacral;

c: linha entre o centro da cabeça femoral e o ponto médio sacral;

IP: incidência pélvica.

Figura 1 - Mensuração da incidência pélvica (IP). 


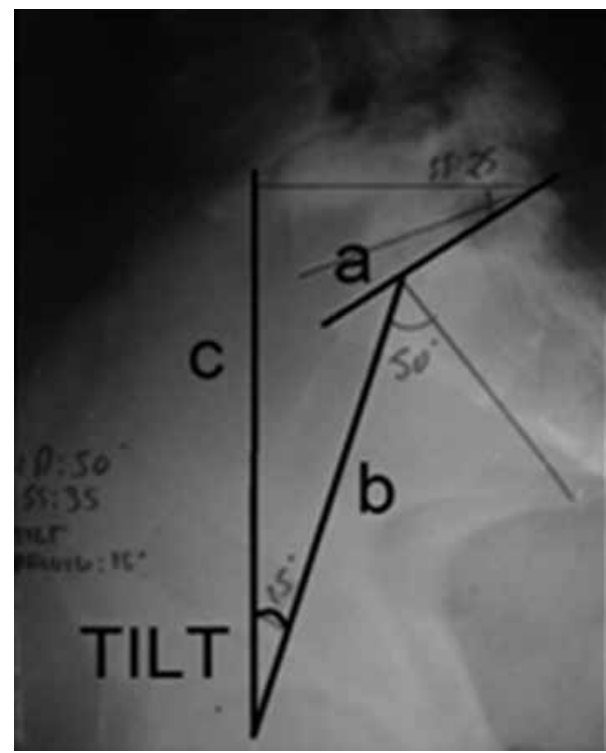

a: placa sacral;

b: linha entre 0 centro da cabeça femoral e o ponto médio sacral;

c: linha de referência no plano vertical;

TILT/PT: inclinação pélvica.

Figura 2 - Mensuração da inclinação pélvica (TILT).

O SLOP sacral, também considerado um parâmetro posicional, é o ângulo entre a placa terminal do sacro (S1) e uma linha de referência num plano horizontal ${ }^{14-16}$ (Figura 3).

Utilizamos o teste Kolmogorov-Smirnov de normalidade dos dados para as variáveis, com a finalidade de

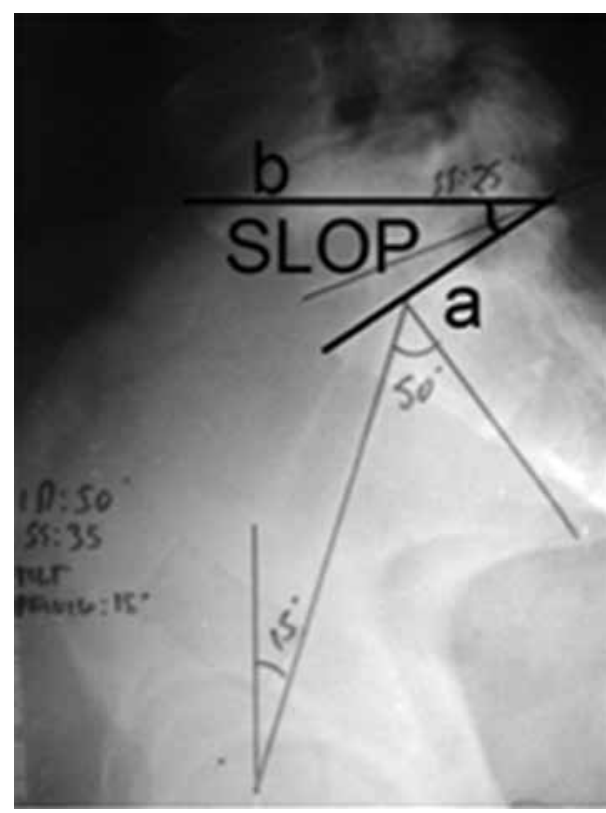

a: placa sacral;

b: linha de referência no plano horizontal;

SLOP/SS: inclinação sacral.

Figura 3 - Mensuração da inclinação sacral (SLOP). avaliar a viabilidade e utilização do teste $t$ de student para análise estatística. Utilizamos também o teste de correlação de Pearson. A análise estatística dos dados foi realizada utilizando os softwares Microsoft Office/Excel 2010 e Statistical Package for the Social Sciences (SPSS).

Os resultados foram numericamente representados por meio de valores da média \pm desvio-padrão, além de alguns dados expressos também em porcentagem. Valores de $\mathrm{p}<0,05$ foram considerados estatisticamente significantes.

\section{Resultados}

No que diz respeito aos parâmetros do equilíbrio espinopélvico, o SLOP sacral e o TILT pélvico tiveram seus extremos de valores variando de 28 a $47^{\circ}$ e de 10 a $42^{\circ}$, respectivamente. Já a IP pélvica variou de 48 a $80^{\circ}$ (Figura 4 ).
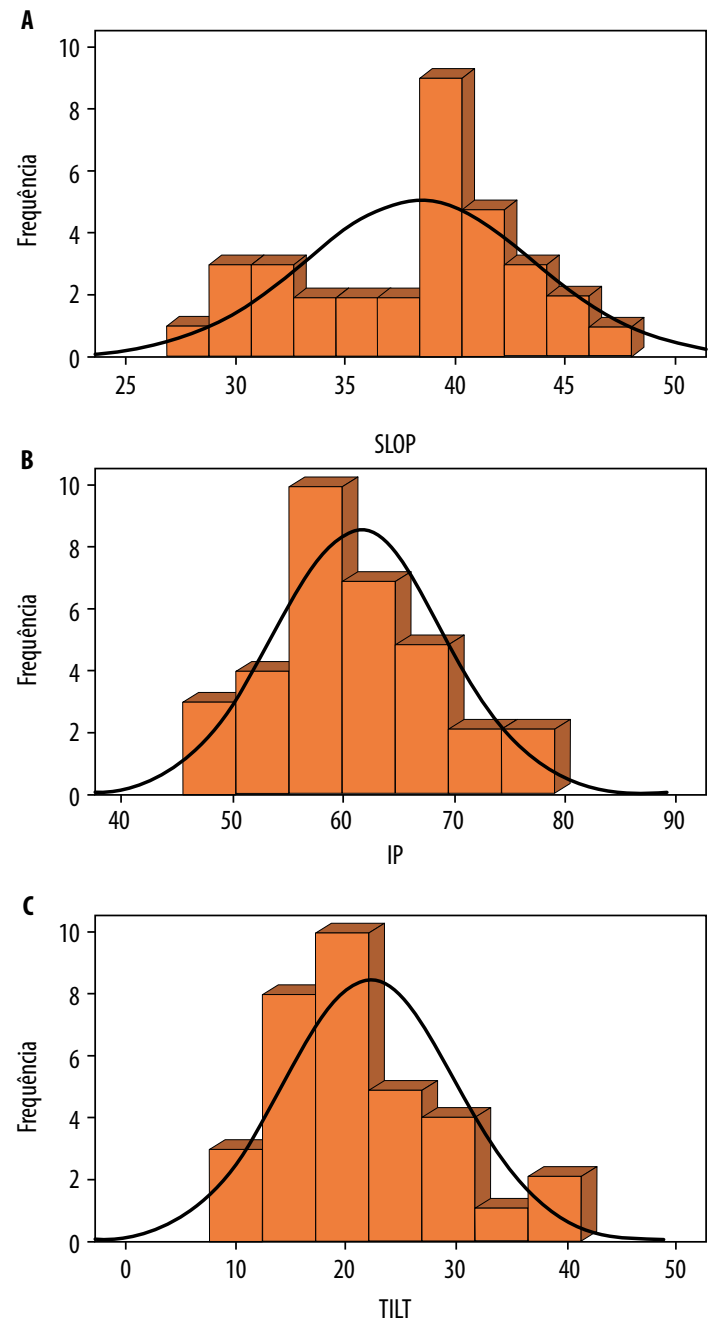

Figura 4 - Distribuição dos parâmetros pélvicos na população em estudos. 
A média da IP foi $61,4^{\circ}$, do SLOP foi $38,3^{\circ}$ e a do TILT, 22, $1^{\circ}$. A análise do desvio-padrão foi mais expressiva na variável TILT, quando comparados com a IP e o SLOP, demonstrando haver maior variabilidade angular nessa variável, conforme a tabela 1 .

Consideramos os resultados das amostras independentes e de variâncias comuns, por indicação do teste preliminar de Levene's de comparação de variâncias, $o$ que permitiu a utilização do teste $t$ de Student.

Ao analisarmos e compararmos as médias da IP, TILT e SLOP, verificamos que há uma correlação positiva entre IP e TILT, ou seja, quanto maior a IP maior será o TILT e uma correlação negativa entre SLOP e TILT, mostrando que quanto maior o SLOP menor será o TILT, ambos com significância estatística conforme a tabela 2 .

\begin{tabular}{|c|c|c|c|}
\hline \multicolumn{4}{|c|}{$\begin{array}{c}\text { Tabela } 1 \text { - Média dos parâmetros envolvidos } \\
\text { no equilíbrio espinopélvico }\end{array}$} \\
\hline $\begin{array}{l}\mathrm{N}^{\circ} \text { pacientes } \\
(\mathrm{N})\end{array}$ & IP & SLOP & TILT \\
\hline 33 & $61,4^{\circ}$ & $38,3^{\circ}$ & $22,1^{\circ}$ \\
\hline Desvio-padrão & $7,7^{\circ}$ & $5,2^{\circ}$ & $7,8^{\circ}$ \\
\hline
\end{tabular}

IP: incidência pélvica; SLOP: inclinação sacral; TILT: inclinação pélvica.

\begin{tabular}{lcc}
\hline \multicolumn{2}{c}{$\begin{array}{c}\text { Tabela } 2 \text { - Correlação entre as variáveis } \\
\text { do equilíbrio espinopélvico }\end{array}$} \\
\hline Tipo de variável & $\begin{array}{c}\text { Correlação de } \\
\text { Pearson }\end{array}$ & $\begin{array}{c}\text { Grau de significância } \\
(\mathbf{2}-\text { tailed })\end{array}$ \\
\hline IP X TILT & $0,618^{* *}$ & 0,000 \\
SLOP x TILT & $-0,400^{*}$ & 0,021 \\
SLOP x IP & 0,180 & 0,316 \\
\hline
\end{tabular}

* A correlação é significativa no nível de 0,05 (2-tailed).

** A correlação é significativa no nível de 0,01 (2-tailed).

\section{Discussão}

No que diz respeito a $\mathrm{ED}$, diversas variáveis anatômicas estão relacionadas ao seu desenvolvimento e progressão, como os parâmetros do equilíbrio espinopélvico (SLOP, TILT e IP) e os fatores individuais de cada paciente como sexo, índice de massa corpórea (IMC), fatores hormonais, hiperfrouxidão ligamentar, artrose lombar, dentre outros. ${ }^{17,18}$

Embora esses fatores sejam conhecidos e relatados em diversos estudos, no que concerne a análise do equilíbrio espinopélvico, o desenvolvimento da ED vem sendo associado a alguns tipos pélvicos específicos que são estudados por meio da IP, TILT e SLOP. ${ }^{17}$

Dentre as variáveis do equilíbrio espinopélvico, a IP é um parâmetro morfológico invariável para cada indivíduo, estando, no entanto, fortemente relacionada com as demais variáveis (SLOP e TILT). ${ }^{17,18}$ A IP é um parâmetro que se mantém estável, independente da posição do paciente durante a realização da radio- grafia. Já o TILT e SLOP são parâmetros que mudam pela posição dos pacientes, sendo o somatório desses equivalentes a IP.

No presente estudo, assim como na literatura, verificamos maior prevalência de ED no sexo feminino, ${ }^{6-9,17,18}$ embora não se tenha uma comparação desta com um grupo controle para avaliar a prevalência global nessa população.

No que tange a IP, Schuller et al., ${ }^{18}$ Barrey et al. ${ }^{19}$ e Morel et al..$^{20}$ demonstraram que pacientes com ED apresentam IP acima dos valores considerados normais para a população, resultados esses semelhantes ao nosso estudo, que encontrou uma média de IP de $61,4^{\circ}$.

Quanto ao SLOP sacral, existem divergências da literatura, uma vez que encontramos uma média de $38,3^{\circ}$, sendo esta inferior às encontradas nos estudos de Barrey et al..$^{19}\left(40,1^{\circ}\right)$ e Schuller et al..$^{18}\left(42,3^{\circ}\right)$, mas superior as de Morel et al..$^{20}\left(37,0^{\circ}\right)$ e Funao et al..$^{17}\left(34,0^{\circ}\right)$.

Os trabalhos de Schuller et al..$^{18} \mathrm{e}$ Funao et al. ${ }^{17}$ avaliaram os parâmetros supracitados comparando as médias encontradas nos portadores de ED com um grupo controle e verificaram que estas são maiores nos grupos de ED, relacionando-as como possíveis fatores de risco para progressão desta, corroborando com o trabalho de Aono et al. ${ }^{21}$ em que sugere que o desenvolvimento e a progressão da ED podem estar relacionados a valores crescentes da IP.

Em relação ao TILT pélvico, o trabalho de Funao et al. ${ }^{17}$ não demonstrou significância estatística quando comparado com o grupo controle. Em contrapartida, Schuller et al. ${ }^{18}$ evidenciaram aumento significativo do TILT em relação ao grupo controle, e no presente trabalho a média geral do TILT foi menor que a dos autores citados anteriormente.

Acreditamos que a variabilidade dos parâmetros TILT e SLOP encontrados nos diversos trabalhos avaliados se deve por uma não padronização e acompanhamento na realização dos exames, feitos em diferentes centros diagnósticos e por diferentes avaliadores.

Para Schuller et al. ${ }^{18} \mathrm{e}$ Funao et al. ${ }^{17} \mathrm{o}$ aumento progressivo do TILT pélvico nos pacientes com altos valores de IP pode ser considerado um mecanismo compensatório na tentativa de restabelecer o equilíbrio espinopélvico, o que vem confirmar a correlação positiva encontrada entre IP e TILT em nosso trabalho, uma vez que essa maior inclinação ocorre com o intuito de se diminuir a pressão intradiscal e consequentemente a dor lombar.

\section{Conclusão}

Nos portadores de ED L4-L5, os parâmetros da IP, TILT e SLOP tiveram suas médias iguais a $61,4^{\circ}, 22,1^{\circ}$ 
e $38,3^{\circ}$, respectivamente, revelando uma alteração na conformação pélvica que culminou em um desequilíbrio espinopélvico nesses pacientes.

\section{Conflitos de interesse}

Os autores declaram não haver conflitos de interesse.

\section{Referências}

1. Junghanns H. Spondylolisthesis ohn spalt in zwischengelenstuck. Arch Orthop Unfallchir. 1930; 29:118-27.

2. Syrmou E, Tsitsopoulos PP, Marinopoulos D, Tsonidis C, Anagnostopoulos I, Tsitsopoulos PD. Spondylolysis: a review and reappraisal. Hippokratia. 2010;14(1):17-21.

3. Kleinstueck FS, Fekete TF, Mannion AF, Grob D, Porchet F, Mutter $\mathrm{U}$, et al. To fuse or not to fuse in lumbar degenerative spondylolistesis: do baseline symptoms help provide the answer?. Eur Spine J. 2012;21(2):268-75.

4. Denard PJ, Holton KF, Miller J, Fink HA, Kado DM, Yoo $\mathrm{JU}$, et al. Lumbar spondylolisthesis among elderly men: prevalence, correlates, and progression. Spine (Phila Pa 1976). 2010;35(10):1072-8.

5. Wiltse LL, Newman PH, Macnab I. Classification of spondylolisis and spondylolisthesis. Clin Orthop Relat Res. 1976;(117):23-9.

6. Rosenberg NJ. Degenerative spondylolisthesis. Predisposing factors. J Bone Joint Surg Am. 1975;57(4):467-74.

7. Fitzgerald JA, Newman PH. Degenerative spondylolisthesis. J Bone Joint Surg Br. 1976;58(2):184-92.

8. Ha KY, Chang CH, Kim KW, Kim YS, Na KH, Lee JS. Expression of estrogen receptors of the facet joints in degenerative spondylolisthesis. Spine (Phila Pa 1976). 2005;30(5):562-6.

9. Devine JG, Schenk-Kisser JM, Skelly AC. Risk factors for degenerative spondylolisthesis: a systematic review. Evid Based Spine Care J. 2012;3(2):25-34.

10. Kong CG, Park JS, Park JB. Sacralization of L5 in radiological studies of degenerative spondylolisthesis at L4-L5. Asian Spine J. 2008;2(1):34-7.
11. Cho BY, Murovic JA, Park J. Imaging correlation of the degree of degenerative L4-5 spondylolisthesis with the corresponding amount of facet fluid. J Neurosurg Spine. 2009;11(5):614-9.

12. Watters WC 3rd, Bono CM, Gilbert TJ, Kreiner DS, Mazanec DJ, Shaffer WO, et al. An evidence-based clinical guideline for the diagnosis and treatment of degenerative lumbar spondylolisthesis. Spine J. 2009;9(7):609-14.

13. Lamartina C, Berjano P, Petruzzi M, Sinigaglia A, Casero G, Cecchinato $\mathrm{R}$, et al. Criteria to restore the sagittal balance in deformity and degenerative spondylolisthesis. Eur Spine J. 2012;21(Suppl 1):S27-31.

14. Mehta VA, Amin A, Omeis I, Gokaslan ZL, Gottfried ON. Implications of spinopelvic alignment for the spine surgeon. Department of Neurosurgery. 2012;70(3):707-21.

15. Kim MK, Lee SH, Kim ES, Eoh W, Chung SS, Lee CS. The impact of sagittal balance on clinical results after posterior interbody fusion for patients with degenerative spondylolisthesis: a pilot study. BMC Musculoskelet Disord. 2011;12-69.

16. Vaz G, Roussouly P, Berthonnaud E, Dimnet J. Sagital morphology and equilibrium of pelvis and spine. Eur Spine J. 2002;11(1):80-7.

17. Funao $\mathrm{H}$, Tsuji $\mathrm{T}$, Hosogane $\mathrm{N}$, Watanabe $\mathrm{K}$, Ishii $\mathrm{K}$, Nakamura M, et al. Comparative study of spinopelvic sagittal alignment between patients with and without degenerative spondylolisthesis. Eur Spine J. 2012;21(11):2181-7.

18. Schuller S, Charles YP, Steib J-P. Sagittal spinopelvic alignment and body mass index in patients with degenerative spondylolisthesis. Eur Spine J. 2011;20(5):713-9.

19. Barrey C, Jund J, Perrin G, Roussouly P. Spinopelvic alignment of patients with degenerative spondylolisthesis. Neurosurgery. 2007;61(5):981-6.

20. Morel E, Ilharreborde B, Lenoir T, Hoffmann E, Vialle $R$, Rillardon L, et al. Sagittal balance of the spine and degenerative spondylolihtesis. Rev Chir Orthop Reparatrice Appart Mot. 2005;91(5):615-26.

21. Aono K, Kobayashi T, Jimbo S, Atsuta Y, Matsuno T. Radiographic analysis of newly developed degenerative spondylolisthesis in a mean twelve-year prospective study. Spine (Phila Pa 1976). 2010;35(8):887-91.

\section{Endereco para correspondência}

Charbel Jacob Júnior

Serviço de Coluna Vertebral,

Hospital Santa Casa de Misericórdia de Vitória

Rua Doutor João Santos Neves, 143, Vila Rubim

29018-180 - Vitória, ES, Brasil

E-mail: jcharbel@gmail.com 